\title{
Coherent and radiative couplings through two-dimensional structured environments
}

\author{
F. Galve* and R. Zambrini ${ }^{\dagger}$ \\ IFISC (UIB-CSIC), Instituto de Física Interdisciplinar y Sistemas Complejos, E-07122 Palma de Mallorca, Spain
}

(Received 1 December 2017; published 23 March 2018)

\begin{abstract}
We study coherent and radiative interactions induced among two or more quantum units by coupling them to two-dimensional (2D) lattices acting as structured environments. This model can be representative of atoms trapped near photonic crystal slabs, trapped ions in Coulomb crystals, or to surface acoustic waves on piezoelectric materials, cold atoms on state-dependent optical lattices, or even circuit QED architectures, to name a few. We compare coherent and radiative contributions for the isotropic and directional regimes of emission into the lattice, for infinite and finite lattices, highlighting their differences and existing pitfalls, e.g., related to long-time or large-lattice limits. We relate the phenomenon of directionality of emission with linear-shaped isofrequency manifolds in the dispersion relation, showing a simple way to disrupt it. For finite lattices, we study further details such as the scaling of resonant number of lattice modes for the isotropic and directional regimes, and relate this behavior with known van Hove singularities in the infinite lattice limit. Furthermore, we export the understanding of emission dynamics with the decay of entanglement for two quantum, atomic or bosonic, units coupled to the 2D lattice. We analyze in some detail completely subradiant configurations of more than two atoms, which can occur in the finite lattice scenario, in contrast with the infinite lattice case. Finally, we demonstrate that induced coherent interactions for dark states are zero for the finite lattice.
\end{abstract}

DOI: 10.1103/PhysRevA.97.033846

\section{INTRODUCTION}

Engineering the coherent interaction between many quantum units is a key ingredient to simulate quantum phases of many-body systems, whereas radiative interactions is instrumental to producing dark states and generating nonclassical states of light. One of the most advanced platforms for quantum simulators are, e.g., two-dimensional (2D) Coulomb crystals of trapped ions [1-3], with vibrational quanta of the ions' motion being used as a channel to effectively induce spin-spin interactions with tunable distance dependence. Excellent control of interactions between atoms and light in one-dimensional (1D) waveguides has been recently shown [4], leading to observation of many atoms superradiance [5]. This has spurred theoretical proposals to use the engineered properties of light to mediate strong long-range atom-atom interactions both in 1D [6] and 2D photonic crystal (PC) lattices [7-9]. Surface acoustic waves (SAW) on piezoelectric materials [10] have been recently proposed as mediators between, e.g., quantum dots, trapped ions, nitrogen-vacancy centers, or superconducting qubits. Control of mediators, be it phonons in the ion and SAW cases, or photons in the PC case, is thus of key importance to shape interactions to build future quantum simulators, as recently proposed for many-body physics [6].

Structured environments, in addition to their usefulness to shape coherent interactions, are a good playground to understand effects of collective dissipation (radiative couplings) of distant units depending on the properties of the substrate. Understanding decoherence and dissipation in realistic scenarios

\footnotetext{
*fernando@ifisc.uib-csic.es

${ }^{\dagger}$ roberta@ifisc.uib-csic.es
}

is of utter importance for the successful realization of quantum technologies. This is, for example, the case when miniaturizing ion traps [11] below the micrometer level, where details of decoherence sources, such as possible correlation lengths among adatom dipoles [12,13], could become accessible [14]. Architectures of two or more units dissipating collectively can lead to super- or subradiance $[15,16]$ and allow certain degrees of freedom to become noiseless $[17,18]$, which is of interest to realizing noise-free operations. Radiative coupling between bosonic probes enabling collective dissipation have been reported in cubic or triangular lattices, either isotropically decaying or purely directional and long range [19]. This translates directly into sub- and super-radiant dynamics, as compared to independent decay rates (no radiative interaction) between quantum units. Long-range radiative couplings not only expand the toolbox of quantum optics, but have important practical implications for quantum networking [20,21] or quantum memories [22-24]. Furthermore, understanding decoherence and dissipation in a broader class of platforms is of utter importance for the successful realization of quantum technologies, where environment microscopic details play a key role, such as, for instance, for miniaturized ion traps [11-13].

Periodic structures can be described as tight-binding models for photons or phonons, disregarding the specific details of the materials used, and so allowing to study fundamental features. We focus on 2D structures because they allow richer features than 1D lattices and can be implemented in PC lattices [7], three-dimensional (3D) arrays of (evanescently coupled) light waveguides whose propagation direction $z$ represents the flow of simulated time [25,26], 2D Coulomb crystals of trapped ions [1-3], or superconducting circuits [27-30] to name a few.

In this work we characterize coherent and radiative couplings induced by a $2 \mathrm{D}$ periodic (squared lattice) structure, both 
infinite and finite, highlighting their similarities and differences. The paper is organized as follows: In Sec. I we introduce the model and its description in terms of a time-dependent master equation for the infinite-lattice case, where we show the behavior of coherent and radiative contributions for isotropic and directional regimes. We analyze finite-time coefficients, explaining their meaning, and also the relation of long-time decay rates with known van Hove singularities [31-33]. We analyze the phenomenon of directional emission, showing how it can be broken, and highlight some pitfalls related to the ordering of large-lattice and long-time limits. An analysis on the validity of the infinite-lattice limit is given, showing it is not advisable to use it for realistic situations. The decay dynamics of entanglement between atomic or bosonic units due to the 2D structure is then studied, showing that it maps with the behavior expected from the prediction of the master equation. In Sec. II we introduce the finite lattice and analyze how the divergence of decay rates due to van Hove singularities is related to the number of resonant modes in the lattice as a function of time. We study how to construct dark states of two, three, and four atoms from the form of the dissipation matrix and show that the form of the Lamb-shift matrix leaves dark states invariant. We finish by comparing exact dynamics of two-atom entanglement in the finite-lattice case with the evolution under the master equation with time-dependent coefficients highlighting the inequivalence between the long-time master equation, timedependent master equation, and exact dynamics. We thus have analyzed many details and possible pitfalls that were absent in previous works in the literature.

\section{INFINITE LATTICE}

We start by analyzing the interaction of a two-unit, bosonic or atomic, quantum system coupled to a structured substrate such as that of Fig. 1 (there a PC lattice is represented). Our analysis holds for periodic, bosonic, linear lattices (modeled by quadratic Hamiltonians). For a square-lattice symmetry dispersion is either (we take the lattice spacing $a=1$ from now on)

$$
\omega_{\vec{k}}=\omega_{0}-2 J\left(\cos k_{x}+\cos k_{y}\right)
$$

for coupled cavities or tight-binding models, or

$$
\omega_{\vec{k}}=\sqrt{\omega_{0}^{2}+8 J\left(\sin ^{2} \frac{k_{x}}{2}+\sin ^{2} \frac{k_{y}}{2}\right)}
$$

for harmonic (springlike) couplings [34]. It can be seen that both dispersions have almost the same shape if they are scaled properly to fit in the range of values. The tight-binding dispersion ranges from $\omega_{0}-4 J$ to $\omega_{0}+4 J$, whereas the harmonic one ranges from $\omega_{0}$ to $\sqrt{\omega_{0}^{2}+16 J}$, so when we use the latter we set $J=3 \omega_{0}^{2} / 16$ so that $\omega_{\vec{k}} \in[1,2] \omega_{0}$ (as in Fig. 1). Of course, we can offset all energies so that the frequency band lies, e.g., at $\omega_{\vec{k}} \in[-1,1] \omega_{0}$ for both dispersions, the only important factors being the detuning between emitters and the resonant pseudomomentum manifold in the lattice. The cases we study in this paper behave equally for both dispersions, because the implied resonant manifolds are the same, so we will interchangeably use both, making the choice explicit only when we use the tight-binding one.

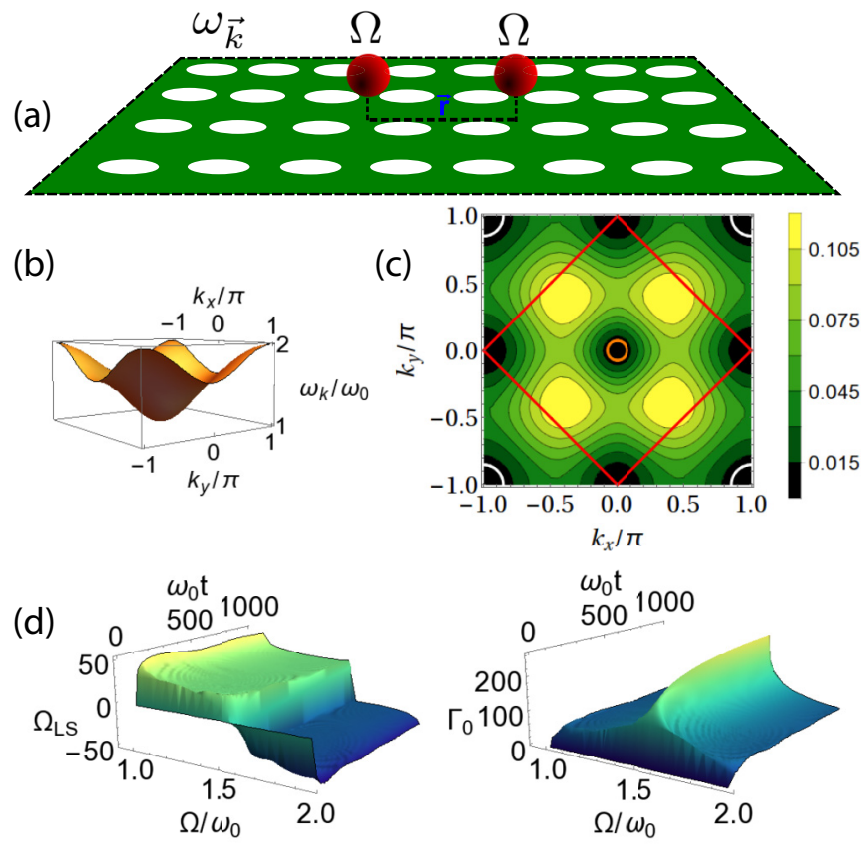

FIG. 1. (a) Sketch of a periodic structured 2D lattice, e.g., a PC. The system is either two harmonic or two-level probes with energy splitting $\Omega$ which couple locally to the structure at a relative distance $\vec{r}$. (b) Lattice's (harmonic) dispersion relation $\omega_{\vec{k}}$, which regulates which manifold of excitations $\vec{k}_{\Omega}$ the two dissipative units couple to. (c) Group velocity with $\omega_{0}=1$ and $J=3 \omega_{0}^{2} / 16$. We have drawn the isoenergy manifold of resonant photon or phonon momenta $\vec{k}_{\Omega}$ for $\Omega=1.01 \omega_{0}$ (orange, central circle), $\Omega=\sqrt{5 / 2} \omega_{0}$ (red, rhomb), and $\Omega=1.98 \omega_{0}$ (white, outer circles). (d) Lamb-shift correction to the splitting $\Omega_{L S}$ and radiative decay rate $\Gamma_{0}$ induced by the lattice (without the factor $\frac{\lambda^{2}}{(2 \pi)^{2}}$ ), with environment frequencies in the range $\omega_{\vec{k}} \in[1,2] \omega_{0}$.

The two identical and independent emitters can be either bosons, $H_{S}=\Omega\left(a_{1}^{\dagger} a_{1}+a_{2}^{\dagger} a_{2}\right)$, or two-level atomic systems (TLS), $H_{S}=\Omega\left(\sigma_{1}^{+} \sigma_{1}^{-}+\sigma_{2}^{+} \sigma_{2}^{-}\right)$. We consider local coupling to the structure

$$
H_{\text {int }}=\lambda\left(a_{1} A\left(\vec{r}_{1}\right)^{\dagger}+a_{2} A\left(\vec{r}_{2}\right)^{\dagger}+\text { H.c. }\right)
$$

or $H_{\text {int }}=\lambda\left(\sigma_{1}^{-} A\left(\vec{r}_{1}\right)^{\dagger}+\sigma_{2}^{-} A\left(\vec{r}_{2}\right)^{\dagger}+\right.$ H.c. $)$ for TLS. The annihilation operators $A(\vec{r})$ for the light field (atoms interacting with PC) or surface displacement field (SAW case) are evaluated at the positions of the atoms (bosons) $\vec{r}_{1}$ and $\vec{r}_{2}$. Interactions extended in space over a range $\xi$ (instead of local) are known to introduce frequency cutoff functions which limit interaction strength for lattice frequencies above $\sim 1 / \xi$ [19]. The interaction between the system and lattice $\lambda$ is assumed to be weak, allowing for a Born-Markov treatment [35]. The dynamics for the environment at $T=0$ is given by

$$
\dot{\rho}_{S}=-i\left[\tilde{H}_{S}(t), \rho_{S}\right]+\sum_{j, l=1}^{2} \Gamma_{j l}(\vec{r}, t)\left(a_{j} \rho_{S} a_{l}^{\dagger}-\frac{1}{2}\left\{a_{l}^{\dagger} a_{j}, \rho_{S}\right\}\right),
$$

with $\tilde{H}_{S}(t)=H_{S}+H_{L S}(t)$ the Lamb-shift corrected Hamiltonian, $H_{L S}(t)=\Omega_{L S}(t)\left(a_{1}^{\dagger} a_{1}+a_{2}^{\dagger} a_{2}\right)+\lambda_{L S}(\vec{r}, t)\left(a_{1} a_{2}^{\dagger}+\right.$ H.c. $)$ and $\vec{r}=\left|\vec{r}_{1}-\vec{r}_{2}\right|$. We define the cross damping $\Gamma_{c} \hat{=} \Gamma_{12}=\Gamma_{21}$ (this equality holds for reciprocal media, which is the usual 
case) and the self-damping $\Gamma_{0} \hat{=} \Gamma_{11}=\Gamma_{22}$ rates. Using $A(\vec{r})=$ $(2 \pi)^{-1} \int_{-\pi}^{\pi} d^{2} \vec{k} \exp (-i \vec{k} \cdot \vec{r}) A(\vec{k})$ (i.e., infinite lattice), the spatial dependent coefficients are

$$
\begin{gathered}
\lambda_{L S}(\vec{r}, t)=\frac{-\lambda^{2}}{(2 \pi)^{2}} \int_{-\pi}^{\pi} d^{2} \vec{k} \frac{1-\cos \left[t\left(\Omega-\omega_{\vec{k}}\right)\right]}{\Omega-\omega_{\vec{k}}} \cos (\vec{k} \vec{r}), \\
\Gamma_{c}(\vec{r}, t)=\frac{2 \lambda^{2}}{(2 \pi)^{2}} \int_{-\pi}^{\pi} d^{2} \vec{k} \frac{\sin \left[t\left(\Omega-\omega_{\vec{k}}\right)\right]}{\Omega-\omega_{\vec{k}}} \cos (\vec{k} \vec{r}),
\end{gathered}
$$

and the onsite coefficients are just $\Omega_{L S}(t)=\lambda_{L S}(0, t), \Gamma_{0}(t)=$ $\Gamma_{c}(0, t)$. They are the sum of counterpropagating plane waves, weighted by their resonance with $\Omega$. The emission of only one unit is proportional to the same integral (note that due to the $\vec{k} \rightarrow-\vec{k}$ symmetry we can replace cos by an exponential), and thus the spatial features of $\Gamma_{c}$ give a precise idea of the radiation pattern of one emitter. We retain, and analyze later, the time dependence of the coefficients in the master equation, which accounts for the buildup of a communication channel between quantum units [36]. This accounts to having performed Born and first-Markov approximations [35]. Usually in the literature the second-Markov approximation (long-time $t \rightarrow \infty$ limit) is used:

$$
\begin{gathered}
\lambda_{L S}(\vec{r}, \infty)=\frac{-\lambda^{2}}{(2 \pi)^{2}} P V \int_{-\pi}^{\pi} d^{2} \vec{k} \frac{\cos (\vec{k} \vec{r})}{\Omega-\omega_{\vec{k}}} \\
\Gamma_{c}(\vec{r}, \infty)=\frac{2 \lambda^{2}}{(2 \pi)^{2}} \int_{-\pi}^{\pi} d^{2} \vec{k} \delta\left(\Omega-\omega_{\vec{k}}\right) \cos (\vec{k} \vec{r}) .
\end{gathered}
$$

The dynamics for identical uncoupled probes can be diagonalized at all times, in the common and relative coordinates $a_{ \pm}=\left(a_{1} \pm a_{2}\right) / \sqrt{2}$, resulting in two completely independent dynamics:

$$
\dot{\rho}_{S}=\sum_{j= \pm}-i\left[H_{j}, \rho_{S}(t)\right]+\Gamma_{j}(\vec{r}, t)\left(a_{j} \rho_{S} a_{j}^{\dagger}-\frac{1}{2}\left\{a_{j}^{\dagger} a_{j}, \rho_{S}\right\}\right),
$$

with $H_{ \pm}=\left(\Omega+\Omega_{L S} \pm \lambda_{L S}\right) a_{ \pm}^{\dagger} a_{ \pm}$and $\Gamma_{ \pm}=\Gamma_{0} \pm \Gamma_{c}$. The latter expression indicates that those modes can become noiseless when $\Gamma_{c}= \pm \Gamma_{0}$, which is known to lead to preservation of entanglement at long times $[18,19]$. When $\Gamma_{c}=+\Gamma_{0}$ we have $\Gamma_{-}=0$ and thus the mode $a_{-}$is not dissipating, which is typically called a common bath situation (CB). With $\Gamma_{c}=$ 0 both modes dissipate, the separate baths scenario (SB). Otherwise, if $\Gamma_{c}=-\Gamma_{0}$ so we have $\Gamma_{+}=0$ and thus it is the center-of-mass motion, which is noiseless [13,37]. We termed this situation anticommon bath (aCB) [13].

The equivalent expressions for the two-TLS case are obtained by substituting $a_{j} \rightarrow \sigma_{j}^{-}$and $a_{j}^{\dagger} \rightarrow \sigma_{j}^{+}$. The dynamics is then diagonal in the operator basis $\sigma_{ \pm}^{-}=\left(\sigma_{1}^{-} \pm \sigma_{2}^{-}\right) / \sqrt{2}$, which has as fixed points $|00\rangle$ and $\left|\Psi_{-}\right\rangle$for $\mathrm{CB}\left(\Gamma_{c} / \Gamma_{0}=1\right)$ with the definition $\left|\Psi_{ \pm}\right\rangle=(|01\rangle \pm|10\rangle) / \sqrt{2}$. The state $|00\rangle$ is trivial in the sense that the lattice is a $T=0$ reservoir. However, if the system starts with $\left|\Psi_{-}\right\rangle$, entanglement is conserved asymptotically. For the aCB case $\left(\Gamma_{c} / \Gamma_{0}=-1\right)$ it is $\left|\Psi_{+}\right\rangle$ which is conserved, and none is conserved asymptotically when SB $\left(\left|\Gamma_{c}\right|<\Gamma_{0}\right)$.

\section{A. Finite-time coefficients}

The coefficients $\Omega_{L S}$ and $\Gamma_{0}$, equal to their single-unit counterparts, can be seen in Fig. 1. Both settle to constant values rather fast, at $\omega_{0} t \approx 10$, justifying the broadly used long-time-limit master equations. The single-unit decay rate $\Gamma_{0}$ also settles quickly to a constant, except for the specific splitting $\Omega=\sqrt{5 / 2} \omega_{0}$ (from now on we will work with the harmonic dispersion, unless otherwise stated), where it has its highest value (for $J=3 / 16 \omega_{0}^{2}$ ). The decay rate at this particular $\Omega$ is divergent, see Fig. 1(d) right, in the long-time limit $\Gamma_{0}(t \rightarrow \infty) \propto \int_{-\pi}^{\pi} d^{2} \vec{k} \delta\left(\Omega-\omega_{\vec{k}}\right)=\int_{\vec{k}_{\Omega}} d^{2} \vec{k}\left|\vec{\nabla} \omega\left(\vec{k}_{\Omega}\right)\right|^{-1}$. It is the sum of all inverse group velocities [density of states, DoS, see Fig. 1(c)] of the manifold of excitations $\vec{k}_{\Omega}$ resonant with $\Omega$. For $\Omega=\sqrt{5 / 2} \omega_{0}$ (red line) it picks up points (black regions in the plot) where the density of states diverges, known as van Hove singularities [33]. Due to the periodic structure of reciprocal space, these singularities are known to appear generically [38] and lead to logarithmic divergences in 2D. We also note that other points (not relevant for our work) have zero DoS at the extrema of the band; here bound states form and perturbative approaches are no longer valid [39-43].

The most important consequence of retaining timedependent coefficients in the master equation, instead of just using their long-time limits (the usual Markovian master equation), comes from the cross talk. From Figs. 2, 3, and 4 we see that it takes a non-negligible time for the coherent and radiative interactions to become nonzero for distant units. During that time, the two quantum units see independent environments, because $\Gamma_{c}=0$, and only when it becomes nonzero do they start "seeing" a correlated environment. Take, for example, two units with $\Omega=1.01 \omega_{0}$, as in Fig. 2, at a distance $\sim 30$ : before $\omega_{0} t=1000$ they have $\Gamma_{c}=0$ and thus decay independently, whereas from that time on they have $\Gamma_{c} \approx \Gamma_{0} / 3$, leading to $\Gamma_{-} / \Gamma_{+} \approx 1 / 2$. This means that an initial state $\left|\psi_{+}\right\rangle$will decay twice faster than $\left|\psi_{-}\right\rangle$, but only from that time onwards.

If the system frequency is increased reaching the middle of the band, Fig. $3\left(\Omega=\sqrt{5 / 2} \omega_{0}\right)$, it was predicted in $[19,44]$
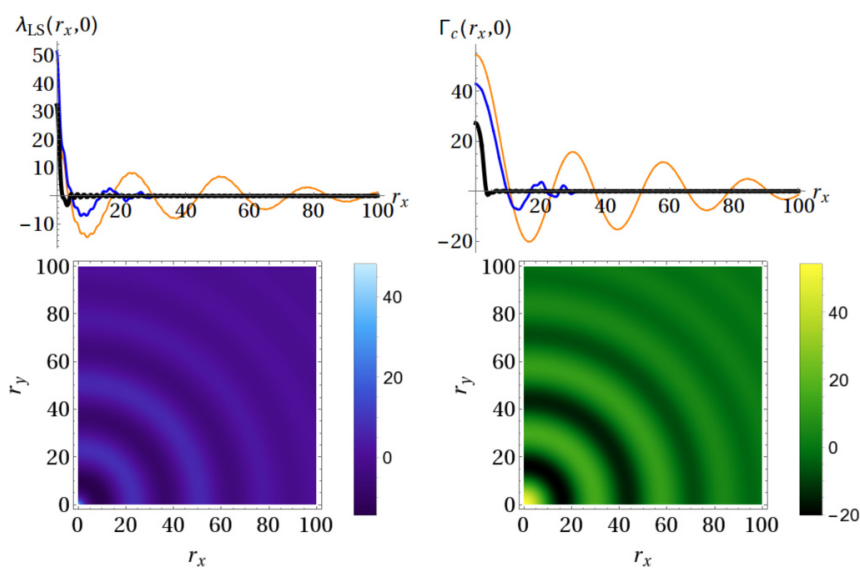

FIG. 2. 2D lattice with $J / \omega_{0}^{2}=3 / 16$ so that $\omega_{\vec{k}} \in\left[\omega_{0}, 2 \omega_{0}\right]$, isotropic case $\Omega=1.01 \omega_{0}$. Top: Lamb-shift coupling (left) $\lambda_{L S}(\vec{r})$ and cross damping (right) $\Gamma_{c}(\vec{r})$, normalized by the factor $\lambda^{2} /\left[(2 \pi)^{2}\right]$, for different times $\omega_{0} t=10$ (black), 100 (blue, gray), 1000 (orange, light gray), along the line $r_{y}=0$. Bottom: Full space dependence of $\lambda_{L S}(\vec{r})$ (left) and $\Gamma_{c}(\vec{r})$ (right) for $\omega_{0} t=1000$. 

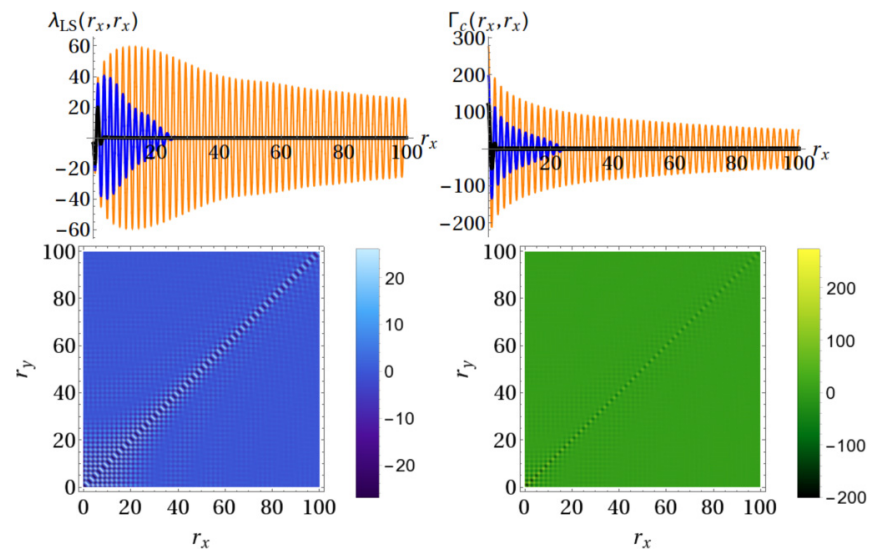

FIG. 3. Same quantities and color codes as in Fig. 2, but for the diagonal case $\Omega=\sqrt{5 / 2} \omega_{0}$. Figures at the top are drawn along the $r_{x}=r_{y}$ line.

that it leads to "diagonal-only" propagation of excitations, and thus to decay of cross damping in all directions except for the diagonals. This behavior has been checked against the exact (atom-lattice case) dynamics (see [9,31,32]).

We plot next the Lamb-shift LS (coherent) coupling $\lambda_{L S}(\vec{r})$ and the cross-damping coefficient $\Gamma_{c}(\vec{r})$, without the irrelevant factor $\lambda^{2} /\left[(2 \pi)^{2}\right]$ for the three cases highlighted in Fig. 1(c), corresponding to increasing frequency of the units interacting with infinite planar squared lattices. In the first case $\left(\Omega \simeq \omega_{0}\right)$ the system is resonant with the low-momentum manifold of excitations in the lattice, corresponding to an approximately isotropic dispersion relation $\omega_{\vec{k}} \simeq \sqrt{\omega_{0}^{2}+2 J|\vec{k}|^{2}}$, as can be seen in the spatial shape of the propagation in Fig. 2. The case of high $k\left(\Omega=1.999 \omega_{0}\right)$ in Fig. 4 resonates with the "corners" of the dispersion relation [Fig. 1(c)] and, although apparently
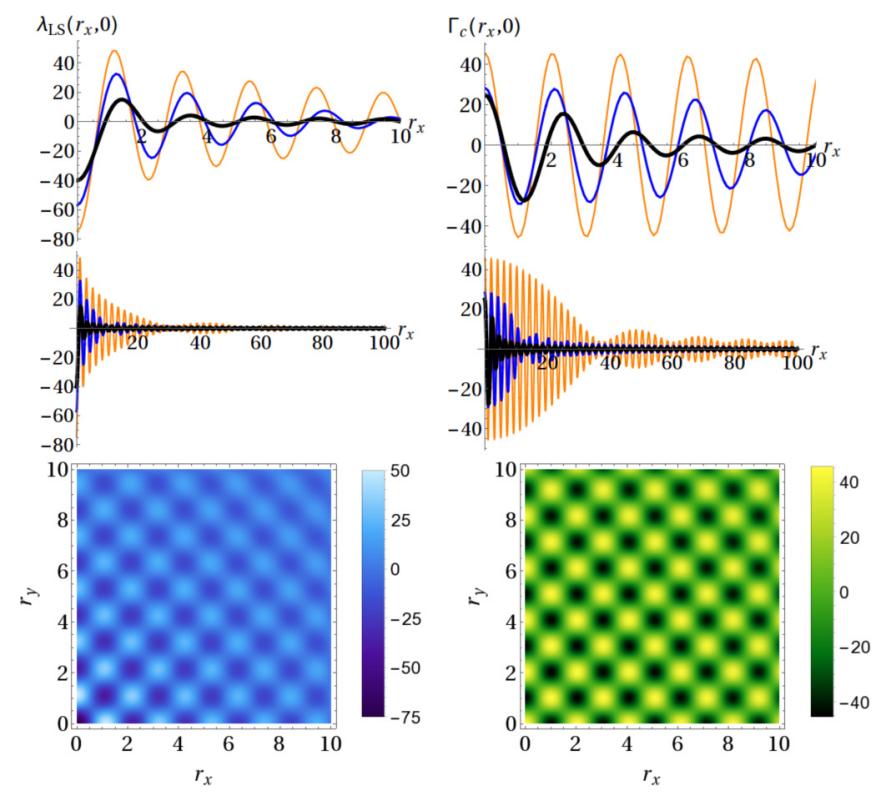

FIG. 4. Same quantities and color codes as in Fig. 2, but for the high- $k$ case $\Omega=1.999 \omega_{0}$. Figures at the top (shorter times) and middle (longer times) are drawn along the $r_{y}=0$ line. anisotropic, can be seen to lead also to isotropic decay of the master equation, with a superimposed alternating oscillation. Writing for one of the four corners $\vec{k}=\left(\pi-\delta_{x}, \pi-\delta_{y}\right)$, we have $\cos (\vec{k} \vec{r})=\cos [\pi(x+y)] \cos \left(x \delta_{x}+y \delta_{y}\right)$. Integration of variables $\delta_{x, y}$ is again isotropic and thus leads to a $J_{0}(|\delta||\vec{r}|)$ decay times the oscillating factor $\cos [\pi(x+y)]$. Finally, the "diagonal" case $\left(\Omega=\sqrt{5 / 2} \omega_{0}\right)$ is a peculiar case where the excitations conspire to produce a purely diagonal propagation [9] as displayed in Fig. 3.

At long times distant points are both coherently and radiatively coupled with different strengths (orange curves respectively in Fig. 2, 3, and 4). It is to be noted that the spatial dependence of coherent and incoherent contributions (for each case) are rather similar although not equal, see, e.g., the beating of a long-time Lamb shift as compared to cross damping (top Fig. 3), or their different decay behaviors (top Fig. 4), since both consist in integrating $\cos (\vec{k} \vec{r})$ but with the different weights $\left[1-\cos \left(\Omega-\omega_{\vec{k}}\right)\right] /\left(\Omega-\omega_{\vec{k}}\right)$ and $\operatorname{sinc}\left(\Omega-\omega_{\vec{k}}\right)$. Both weights are most important when $\Omega \simeq \omega_{\vec{k}}$ but have different functional behavior, leading to these differences.

\section{B. Radiative directional coupling}

The "diagonal" $\left(\Omega=\sqrt{5 / 2} \omega_{0}\right.$ or $\Omega=\omega_{0}$ for the tightbinding dispersion relation) was highlighted in [19] as displaying purely directional radiative coupling (also radiation by one emitter). This happens for cubic and triangular lattices [19], and also their higher-dimensional analogs, as well as for graphene [45], being then present in common geometric configurations. It has been recently explained as a consequence of linear-shape isofrequency manifolds in the dispersion relation [9]: a rotation into that momentum coordinate selects a specific value of that momentum, which produces a plane wave (or sine) in that direction, and is thus nondecaying. In the orthogonal coordinate we still sum many momenta, which gives generically an incoherent sum of waves and thus a decaying function along the corresponding spatial coordinate. A simple mechanism breaking the linear shape for a squaresymmetric lattice was proposed in [9], consisting in adding a hopping term between diagonal neighbors in the lattice. This results in, e.g., a modified tight-binding dispersion relation $\omega_{\vec{k}}=\omega_{0}-2 J\left(\cos k_{x}+\cos k_{y}\right)-4 \tilde{J} \cos k_{x} \cos k_{y}$, whose last term gives curvature to the isofrequency manifold. This is easily seen in Fig. 5.

Because of the van Hove singularities in this manifold, the case of directional emission allows inspection of some possible pitfalls related to the ordering of the large-lattice $(N \rightarrow \infty)$ and long-time limits $(t \rightarrow \infty)$. If we first obtain the analytic shape of $\Gamma_{c}(\vec{r})$ for periodic boundary conditions by taking a finite lattice with $t \rightarrow \infty$ [which exactly selects $N / 2$ modes on each of the red straight lines in Fig. 1(c)] and taking then the large-lattice limit $N \rightarrow \infty$, it yields $\Gamma_{c}(\vec{r}) / \Gamma_{0}=(x \sin \pi x-$ $y \sin \pi y) /\left[\pi\left(x^{2}-y^{2}\right)\right]$, i.e., $[\operatorname{sinc}(\pi|\vec{r}|)+\cos (\pi|\vec{r}|)] / 2$ at the diagonals and $\Gamma_{c}(\vec{r}) / \Gamma_{0}=\operatorname{sinc}\left(\pi r_{y}\right)$ at $r_{x}=0$. This function has a maximum value 1 at $|\vec{r}|=0$ and after the $\operatorname{sinc}(\pi|\vec{r}|)$ decays, it has a maximum value $1 / 2$ at the diagonals and is 0 elsewhere. That is, it is a cross-shaped oscillating function of amplitude $1 / 2$.

It is a peculiar fact that interchanging the limits gives a different result. By setting $t \ll N$ and doing $N \rightarrow \infty$ in fact 

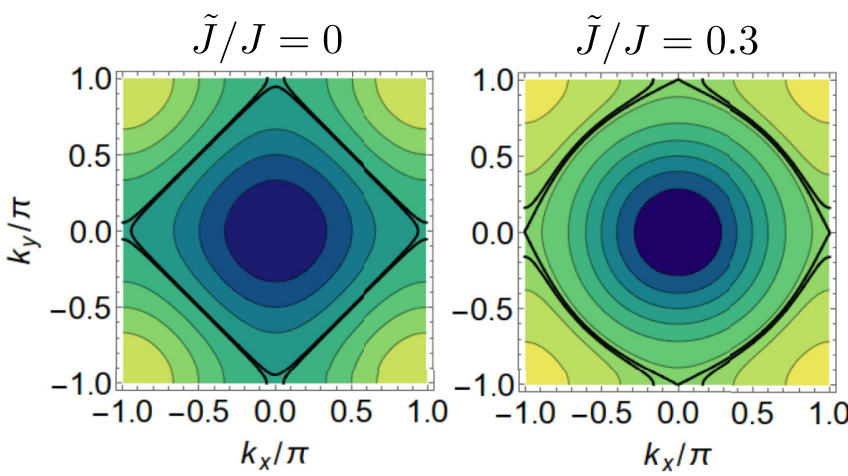

FIG. 5. Tight-binding dispersion relation without $\tilde{J} / J=0.0$ (left) and with $\tilde{J} / J=0.3$ (right) next-to-nearest-neighbor hopping. The manifold of interest, highlighted between darker contour lines, ceases to be straight and acquires a curvature.

gives a maximum value $\Gamma_{c}(x=y) / \Gamma_{0}=1$ even far from the origin. At intermediate $N$ we see a shape similar to directional radiation but with a region near $\vec{r}=0$ with almost maximum cross talk (see Fig. 6), i.e., higher than the $1 / 2$ value obtained when $t \gg N$. In addition, its absolute value is logarithmicdivergent, as is well known from van Hove singularities [33]. The very different behavior can be explained as follows: in the first case, $t \gg N$, only $N / 2$ modes are resonant, because the spacing in momentum space goes as $\Delta k \approx 1 / N$ and $t$ is so large that the $\operatorname{sinc}\left[t\left(\Omega-\omega_{\vec{k}}\right)\right]$ function selects a momentum manifold always smaller than $\Delta k$. In that way, we only sum the contribution of a finite number of modes. In spite of that, see later discussion on the scaling of the decay rate, the cross talk diverges as $N t$. In the second case, it is even worse: for $t \ll$ $N$ the amount of resonant modes selected by the $\operatorname{sinc}[t(\Omega-$ $\left.\left.\omega_{\vec{k}}\right)\right]$ is even bigger, and does not have only contributions from the momentum manifold yielding directionality; it has more contributions, and this is reflected in the modified cross-talk shape in Fig. 6.

\section{Time scales and modes choice}

A comment is in order about time scales. The possibility to use exponential waves as ansatz for guided modes in a lattice totally disregards that lattices are always finite, an assumption that can be perfectly valid within a range of parameters. Take, for example, the tight-binding dispersion and note that the

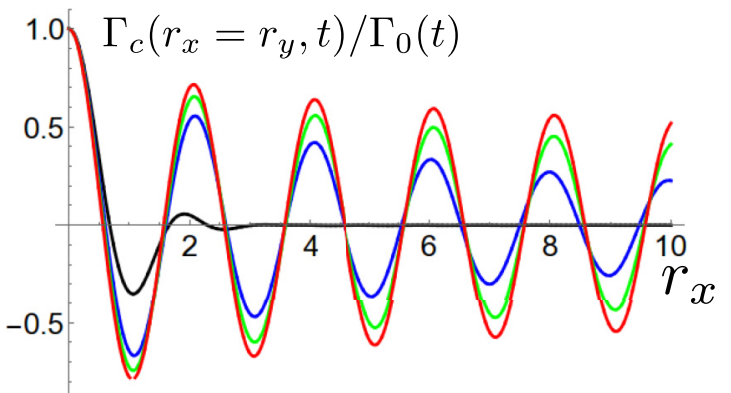

FIG. 6. Normalized cross talk as a function of distance, with $J t / N=10$ (tight-binding dispersion), and $N=20,200,1000,5000$ (colors: black, blue, green, red; with increasing vertical amplitude). highest group velocity $d \omega_{\vec{k}} / d \vec{k}$ [see Fig. 1 (c)] is $2 \sqrt{2} J$. Once any signal reaches the boundary of the lattice, bouncings and revivals of the system dynamics are expected to occur. This happens for times $J t \approx N$. The decay dynamics of the system is however governed by the system-lattice coupling $\lambda$, yielding a decay rate $\Gamma_{0} \approx \lambda^{2}$, and thus significant decay is expected to happen at times $\sim 1 / \lambda^{2}$. Thus the exponential wave ansatz is valid whenever the characteristic decay time is at least smaller than the revival of signals at the boundaries. This implies $1 / \lambda^{2}<N / J$, and thus requires $\lambda^{2}>J / N$. Taking $J$ as energy scale, and a lattice of $N \approx 100$ sites, already implies $\lambda \approx 0.1 J$, within the strong-coupling regime, as in [31]. For this reason it is advisable to use finite-lattice mode functions, as we do in later sections.

\section{Entanglement decay}

We analyze next how an initial entangled state of the two bosonic or TLS probes evolves as a result of the interaction $\mathrm{C}\left(r_{y}=0\right)$

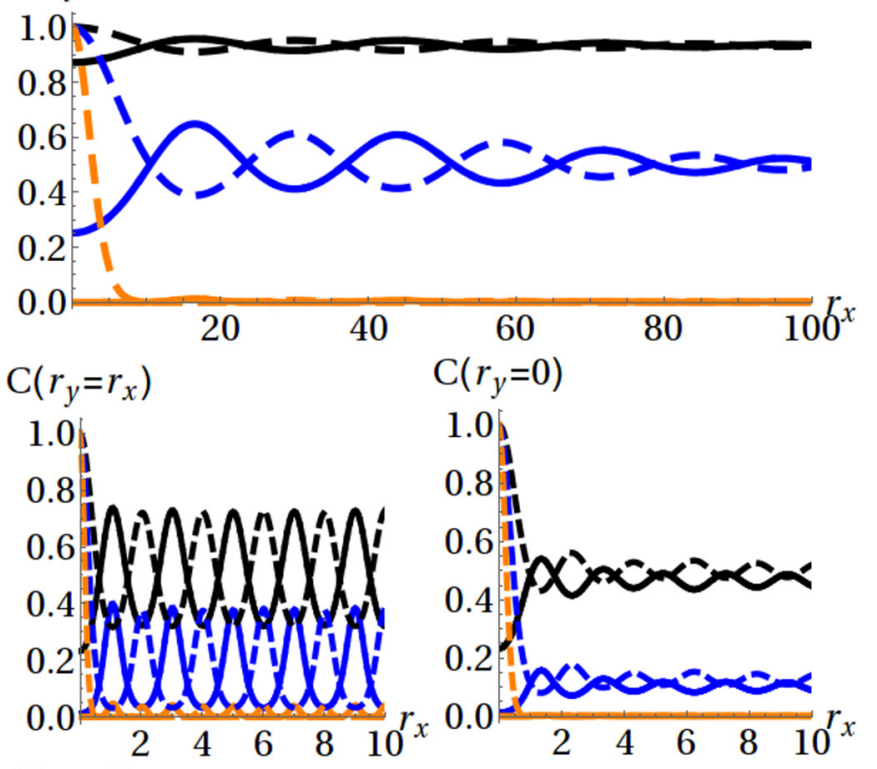
$\mathrm{C}\left(r_{y}=0\right)$

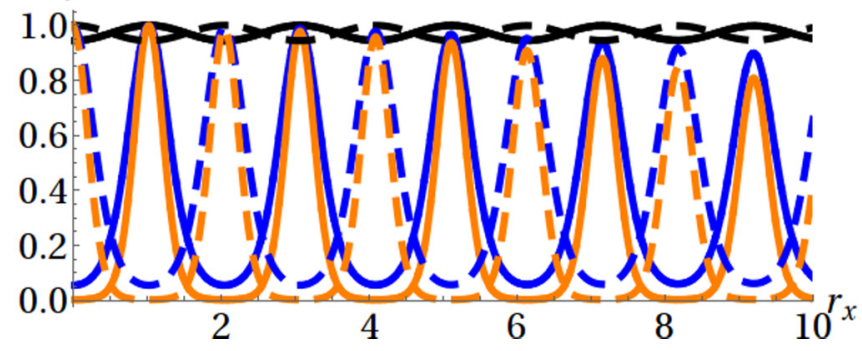

FIG. 7. Spatial dependence of concurrence $C$ when one TLS sits at $\left\{r_{x}, r_{y}\right\}$ distance from the other, with initial state $\left|\Psi_{-}\right\rangle$(dashed lines) or $\left|\Psi_{+}\right\rangle$(continuous lines) possessing maximum entanglement $C=$ 1. The system-environment coupling is $\lambda=0.01 \omega_{0}$. Top: Isotropic case $\Omega=1.001 \omega_{0}$ at times $\omega_{0} t=10^{3}$ (black), $10^{4}$ (blue, gray), $10^{5}$ (orange, light gray). Note that the solid and dashed lines coincide. Middle: "Diagonal" case $\Omega=\sqrt{5 / 2} \omega_{0}$ at times $\omega_{0} t=10^{3}$ (black), $3 \times 10^{3}$ (blue, gray), $10^{4}$ (orange, light gray). Bottom: High- $k$ case $\Omega=1.999 \omega_{0}$ at times $\omega_{0} t=10^{3}$ (black), $5 \times 10^{4}$ (blue, gray), $10^{5}$ (orange, light gray). 

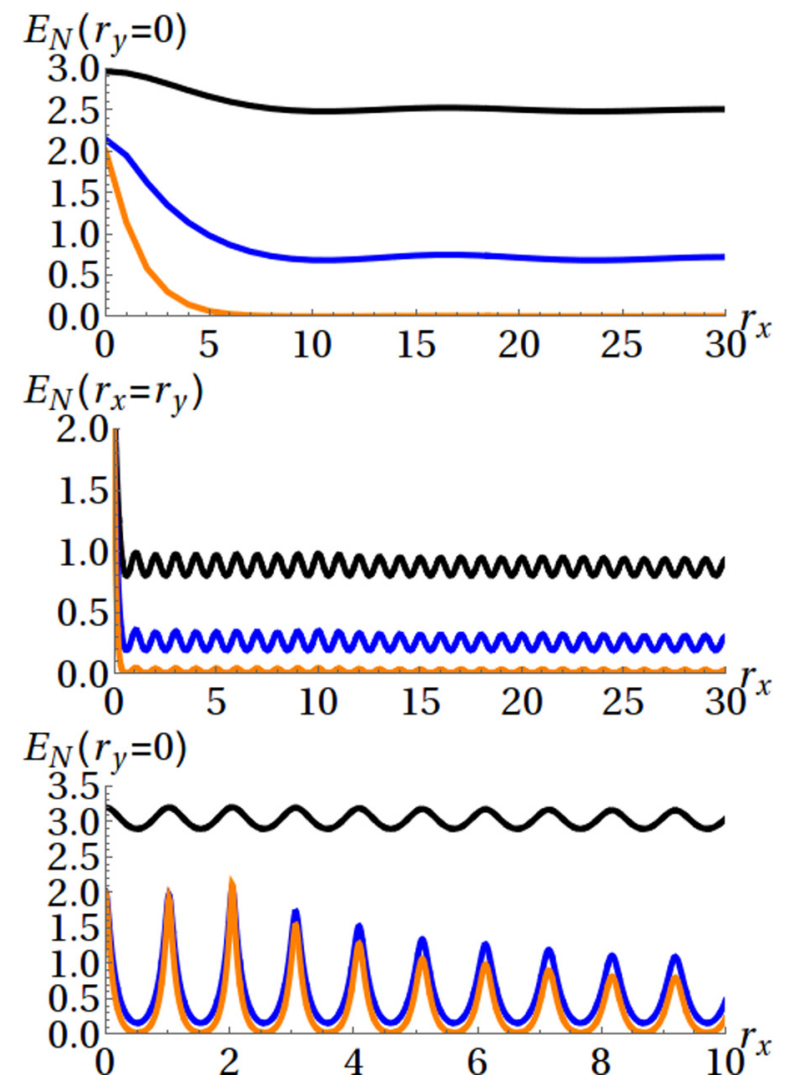

FIG. 8. Spatial dependence of logarithmic negativity $E_{\mathcal{N}}$ when one oscillator sits at $\left\{r_{x}, r_{y}\right\}$ distance from the other, with an initial two-mode squeezed vacuum state with squeezing factor $r=2$; this state has $E_{\mathcal{N}}=4$. The parameters $\lambda, \omega_{0} t$, the color codes, and the cases are as in Fig. 7.

with an infinite lattice. We consider a two-mode squeezed vacuum [46-48] for the oscillator case, and one of the two maximally entangled Bell states $\left|\Psi_{ \pm}\right\rangle=(|01\rangle \pm|10\rangle) / \sqrt{2}$ for the two-TLS case. To quantify entanglement for two TLS, we use the concurrence $C$ [49] and for the bosonic case the logarithmic negativity [50]. We start with the dynamics when the atom-lattice interaction is so weak that the master equation coefficients can be assumed to reach their constant asymptotic values, corresponding physically to the regime where waves between atoms have enough time to become stationary (i.e., first and second Markov approximations). We show results for a couple of TLS for different times $\omega_{0} t$ (to be compared with the system-bath interaction time $\left.1 / \lambda^{2}=10^{4} / \omega_{0}\right)$ and distances $\vec{r}$ in Fig. 7, where we plot the surviving concurrence for initial states $\left|\Psi_{ \pm}\right\rangle$. They follow the qualitative behavior of the cross damping's spatial dependence, including the alternating behavior of $\left|\Psi_{ \pm}\right\rangle$as the sign of $\Gamma_{c}$ changes.

There is noiseless behavior (total subradiance) only if we are able to make $\vec{r} \ll 1 /\left|\vec{k}_{\Omega}\right|$ (low $k$ ) for $\left|\Psi_{-}\right\rangle$, or with $\Omega$ at the high- $k$ edge of the band, placing the TLS at odd $\left(\left|\Psi_{-}\right\rangle\right)$or even $\left(\left|\Psi_{+}\right\rangle\right)$distances from each other. It must be noted, though, that when the units are near the band edges they form atom-photon (or ion-phonon) bound states which cannot be treated under Born-Markov conditions [39-41]. The interesting "diagonal"

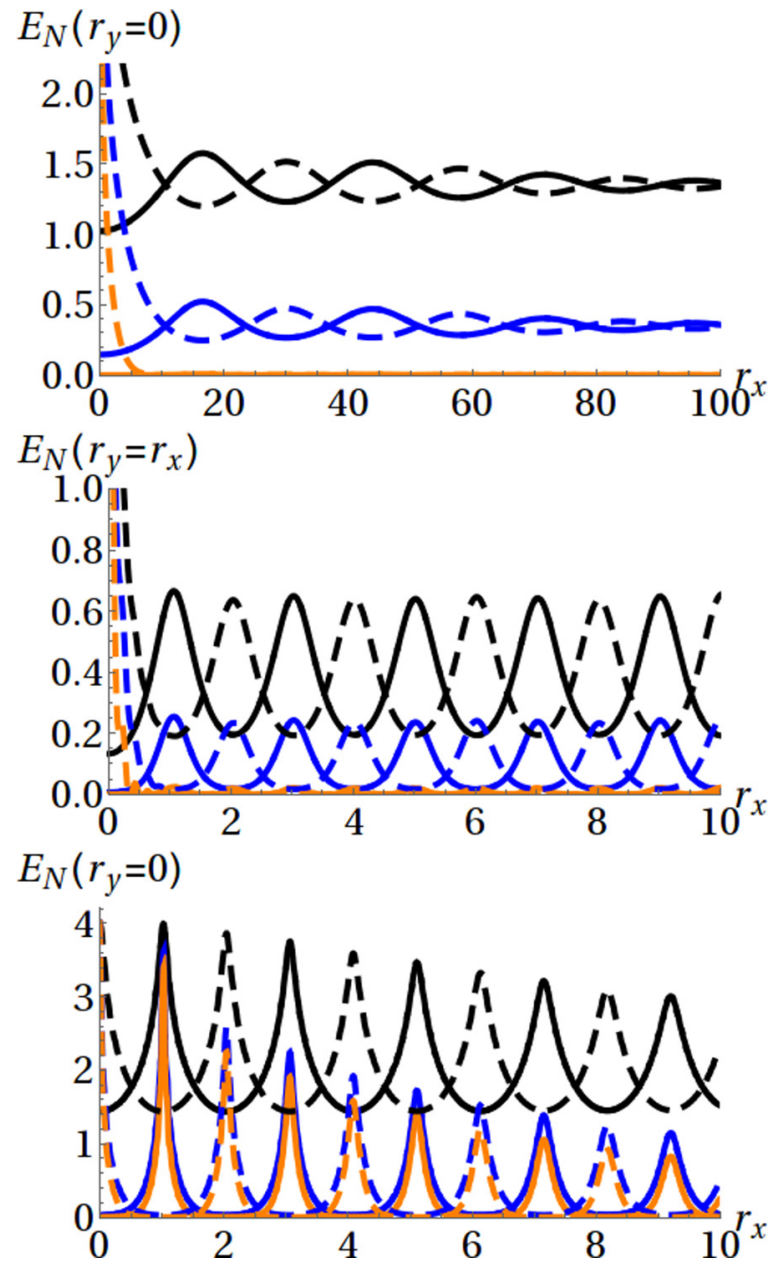

FIG. 9. Same as in Fig. 8, but the initial state is asymmetrically squeezed (see text), with $\left(r_{1}, r_{2}\right)=(4,0)$ (continuous lines) and $\left(r_{1}, r_{2}\right)=(0,4)$ (dashed lines); this state also has $E_{\mathcal{N}}=4$ but behaves more similarly to the atomic Bell states. The parameters $\lambda, \omega_{0} t$, the color codes, and the cases are as in Fig. 7.

case features $\Gamma_{c}(\vec{r}) / \Gamma_{0}=1 / 2$ as maximum values, so it never produces noiseless dynamics.

The case of a bosonic two-mode state is, in comparison, insensitive to the distinction between $\mathrm{CB}$ and $\mathrm{aCB}$, as can be seen in Fig. 8. (In some sense each degree of freedom acts as each Bell state, but now both are present in the two-particle dynamics.) This leads to similar although faster decay. In order to see a behavior similar to atomic Bell states, we need to initialize the state as $|r\rangle_{+} \otimes|0\rangle_{-}$(or $|0\rangle_{+} \otimes|r\rangle_{-}$), the equivalent of $\left|\psi_{+}\right\rangle$(or $\left|\psi_{-}\right\rangle$, respectively), with the mode corresponding to operator $a_{+}$squeezed and the other in vacuum (or vice versa). In comparison, the two-mode squeezed state is $|r\rangle_{+} \otimes|-r\rangle_{-}$. In this asymmetrically squeezed case, the dynamics is qualitatively similar to atomic Bell states, as can be seen in Fig. 9.

\section{FINITE-SIZE LATTICES}

Finite-size PC lattices typically need finite-element calculations to compute the guided modes [51] and are leaky at their boundaries. However, we can use guided modes coupled to the 
atoms: these modes have evanescent tails at the outside of the crystal. Also, we could enforce Dirichlet boundary conditions for all allowed modes (i.e., also guided resonance modes which are within the light cone and thus can interact with the outer modes, allowing extraction of light [52]) with Bragg mirrors at the boundaries [53]. Assuming that in practice this can be achieved with enough precision, traveling waves are then replaced by standing waves,

$$
f_{\vec{n}, \vec{k}}=\frac{2}{N+1} \sin \left(k_{x} n_{x}\right) \sin \left(k_{y} n_{y}\right)
$$

with $k_{x, y}=\pi l_{x, y} /\left(N_{x, y}+1\right), n_{x, y} \in\left[1, N_{x, y}\right]$, and the same for $l_{x, y} . N_{x, y}$ is given by the amount of dielectric function changes in each direction, typically associated with holes made in a dielectric slab, or pillars above it [51]. For the finite-size lattice we will take the tight-binding dispersion relation, which is as before $\omega_{0}-2 J\left(\cos k_{x}+\cos k_{y}\right)$. A unitary transformation of the Hamiltonian removes the irrelevant offset $\omega_{0}$, which from now on we take as zero. Now the damping coefficients become

$$
\begin{aligned}
& \Gamma_{0}=2 \lambda^{2} \sum_{k_{x}, k_{y}=1}^{N} \frac{\sin \left[t\left(\Omega-\omega_{\vec{k}}\right)\right]}{\Omega-\omega_{\vec{k}}}\left|f_{\vec{n}, \vec{k}}\right|^{2}, \\
& \Gamma_{c}=2 \lambda^{2} \sum_{k_{x}, k_{y}=1}^{N} \frac{\sin \left[t\left(\Omega-\omega_{\vec{k}}\right)\right]}{\Omega-\omega_{\vec{k}}} f_{\vec{n}, \vec{k}} f_{\vec{n}^{\prime}, \vec{k}},
\end{aligned}
$$

and accordingly, the LS coherent contributions.

\section{A. Number of resonant modes}

Once a discrete spectrum is assumed, consistent with the finite size of the lattice, one can understand the divergence in the decay rate as a scaling of the number of resonant modes as follows. Let us take for simplicity the case of periodic boundaries, so $f_{\vec{n}, \vec{k}}=e^{-i \vec{k} \cdot \vec{n}} / 2 \pi$ and $\left|f_{\vec{n}, \vec{k}}\right|^{2}=(2 \pi)^{-2}$. The decay rate in this case is

$$
\Gamma_{0}(t)=\frac{2 \lambda^{2} t}{(2 \pi)^{2}} \sum_{\vec{k}} \operatorname{sinc}\left[t\left(\Omega-\omega_{\vec{k}}\right)\right]
$$

For long times, this sum approximately counts how many modes in the crystal are resonant with $\Omega$, because for them the sinc function is 1 , and 0 elsewhere. In Fig. 10 we can see that for frequencies $\Omega \neq 0$, at the beginning all modes are resonant, but as time grows, the number of resonant modes quickly reaches a scaling $t^{-1}$, making the decay rate tend to a constant already for $J t \approx 10$, consistent with Fig. 1(d). This is a common situation, justifying the use of long-time master equations (Markov 2 approximation [35] with constant coefficients). However, for $\Omega=0$ we see that the number of resonant modes scales as $t^{-0.8}$, and thus the decay rate scales as $t^{0.2}$. At long times it saturates at a value $4 \times N / 2$ and does not fall more, because the resonant manifold defined by the red square in Fig. 1(b) $(N / 2$ modes in each of the four lines) perfectly fits the sinc function in Eq. (7), whatever $t$. From this

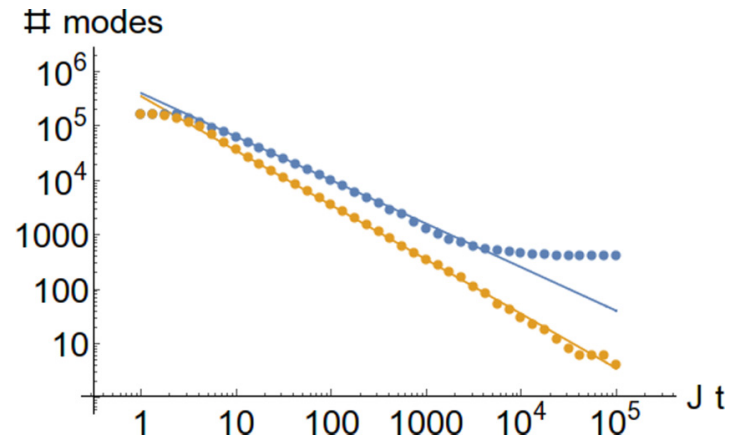

FIG. 10. Number of modes resonant (i.e., selected by the sinc function, with $\Omega=0$ (blue, upper dots) and $\Omega=2 J$ (beige, lower dots) as a function of time with $N=400$. Fits with $t^{-1}$ (beige, lower continuous line) and $t^{-0.8}$ (blue, upper continuous line) are drawn for comparison.

time on, the decay rate scales as $N t$, diverging for $N \rightarrow \infty$ as expected from a van Hove singularity.

\section{B. Multiatom dark states}

Dark states (perfect subradiance) can arise among distant atoms in $1 \mathrm{D}$ but not in $2 \mathrm{D}$ isotropic environments. In the following we show that the "diagonal" case with its linear radiation pattern can be used to construct subradiant states, in analogy with the $1 \mathrm{D}$ case where $\Gamma_{c}^{(1 D)} / \Gamma_{0}=\cos \left(k_{\Omega} x\right)[5,19]$, i.e., it makes $\left|\Psi_{ \pm}\right\rangle$subradiant for specific distances between atoms due to coherent cancellation of waves. It is easy to see geometrically in an open $2 \mathrm{D}$ configuration that, because both atoms radiate with a cross pattern, only one of the lines (the one which joins their positions) can cancel. As noted recently [31,32], no subradiant $2 \mathrm{D}$ configuration of two atoms exists in infinite planar settings. Geometrically again, we see that if we can join the remaining two lines, total cancellation can occur [9]. Indeed, this is the case for reflecting boundaries, as seen in Fig. 11(a). Only four positions for the two atoms will yield total cancellation (equivalently, $\Gamma_{c} / \Gamma_{0}=1$, red points): placing two atoms on any of those points with a state $\left|\Psi_{-}\right\rangle$ yields total subradiance, up to retardation effects. We stress that these dark states can be created at arbitrary distances, depending on the offset from the center of the PC slab.

The reflection of (photonic or phononic) waves can be used to build peculiar radiation patterns of multiunit architectures. In Figs. 11(b)-11(d) we show the cross damping $\Gamma_{c} / \Gamma_{0}$ of one atom with respect to another atom at position $\left(r_{x}, r_{y}\right)$ : one unit (green) shares a partial subradiant condition $\left(\Gamma_{c} / \Gamma_{0}=1 / 2\right)$ with another unit (red), the red unit shares partial subradiance with the blue unit $\left(\Gamma_{c} / \Gamma_{0}=1 / 2\right)$, but the green and blue units radiate independently. We have plotted the cross talk of each of the atoms with the rest of the possible atomic positions in the crystal, to make it more understandable. In this particular case the decay rate matrix would be $\left.\Gamma_{i, j}=\{1,1 / 2,0\},\{1 / 2,1,1 / 2\}\{0,1 / 2,1\}\right\}$, with the slowest decay channel corresponding to state $|\psi\rangle=(|100\rangle-$ $\sqrt{2}|010\rangle+|001\rangle) / \sqrt{2}$ (with atom notation $\mid$ green, red, blue $\rangle$ ) radiating 5.8 and 3.4 slower than the other two orthogonal states of three atoms. 


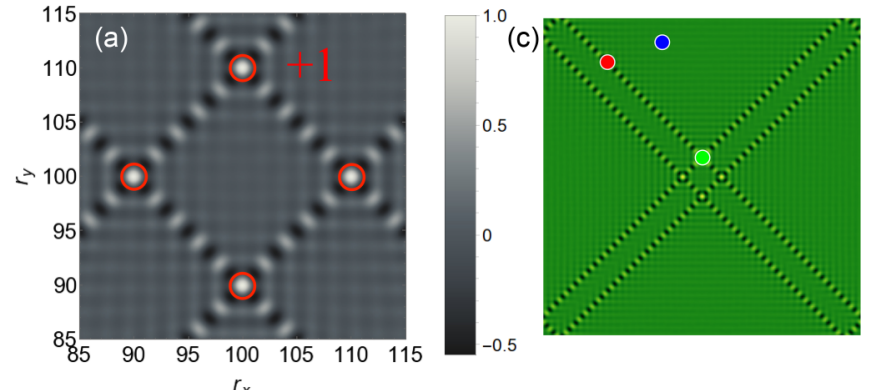

(b)

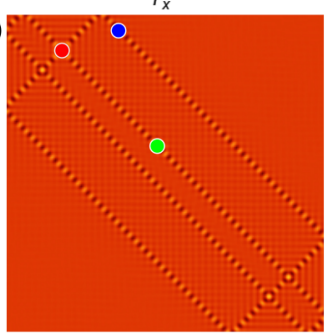

(d)

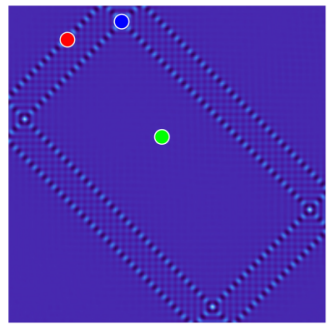

FIG. 11. "Diagonal" case $\Omega=\omega_{0}=0$. (a) Cross damping (in a $200 \times 200$ lattice $)$, e.g., between a particle at $(100,110)$ and another at $\left(r_{x}, r_{y}\right)$. The four points display $\Gamma_{c} / \Gamma_{0}=+1$ (red, drawn circles). If we place one atom in each of these four points we get three completely subradiant and one superradiant states. Case with 3 atoms (in a $100 \times$ 100 lattice): probe 1 is green (center), probe 2 is red (top, left), and probe 3 is blue (top, middle); in (b) we have plotted the cross damping felt between the red particle and another particle sitting at $\left(r_{x}, r_{y}\right)$, i.e., $\Gamma_{c}\left(\right.$ red; $\left.r_{x}, r_{y}\right) / \Gamma_{0}$. In (c) and (d) the same quantity for green and blue particles, respectively.

Another interesting case is to put four atoms, e.g., each in the points highlighted in Fig. 11(c) (rhomboid configuration). This leads to $\Gamma_{i, j}=1, \forall i, j$ and thus to one superradiant state with decay $4 \Gamma_{0},\left|\psi_{\text {super }}\right\rangle=(|1000\rangle+|0100\rangle+|0010\rangle+$ $|0001\rangle) / 2$, or simply $\left|\psi_{\text {super }}\right\rangle=\frac{1}{2}(1,1,1,1)$ (expressed in the basis $\{|1000\rangle,|0100\rangle,|0010\rangle,|0001\rangle\}$, with atom notation $\mid u p$, bottom, left, right $\rangle)$. Also to completely subradiant states of two (e.g., $(1,0,0,-1) / \sqrt{2})$, three $($ e.g., $(-1,0,2,1) / \sqrt{3})$, and four atoms (e.g., $(1,-3,1,1) / 2 \sqrt{3}$; see also [9]). Notice that one can keep several of the atoms in the ground state and they will not affect the subradiance of the remaining excited atoms. In contrast, in infinite lattices the decay rates have the same superradiant state with lower decay rate $2 \Gamma_{0}$, a totally subradiant four-atom state $[31,32]\left|\psi_{1}\right\rangle=\frac{1}{2}(-1,-1,1,1)$, and the states of two or three atoms now radiate with $\Gamma_{0}$. These examples show the potential of this platform to explore the exotic scenarios arising when all sites are filled with atoms in different architectures.

\section{Lamb-shift coupling}

One could worry about the stability of dark states because of the Lamb-shift coupling, which in principle could modify the phases of dark states turning them into bright. For the most interesting "diagonal" case, and in contrast with the infinitelattice scenario, the values of $\lambda_{L S}$ are far smaller than the radiative coupling $\Gamma_{c}$. In spite of both having the same spatial shape (see Fig. 12, now taking the tight-binding dispersion), we observe (not shown) that the ratio $\lambda_{L S} / \Gamma_{c}$ grows linearly with a rate $\sim 10^{-6}$, i.e., it takes $J t \approx 10^{5}$ to reach $\lambda_{L S} / \Gamma_{c} \approx 0.1$.
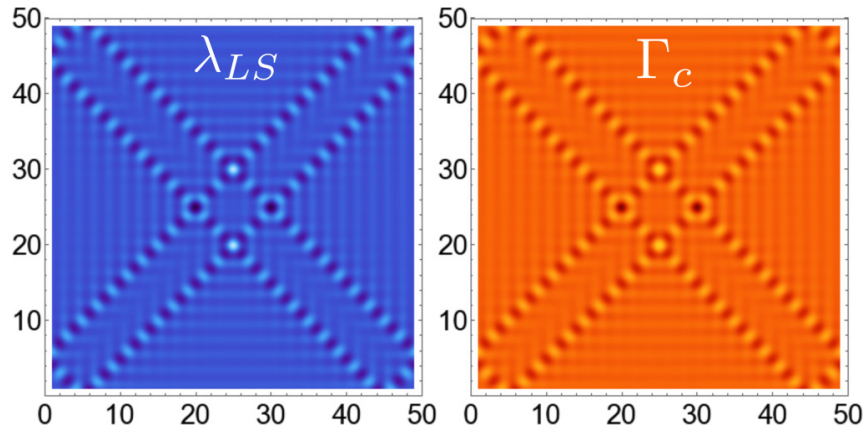

FIG. 12. Comparison of normalized Lamb-shift coupling $\lambda_{L S} / \Omega_{L S}$ (left) and cross talk $\Gamma_{c} / \Gamma_{0}$ (right) between an atom at position $(25,30)$ and another atom at position $\left(r_{x}, r_{y}\right)$ (abscissa and ordinate in the plots, respectively) with a tight-binding lattice $N_{x}=N_{y}=49$, at times $J t=10^{5}$. This corresponds to one of the cases studied in [9]. In absolute values $\lambda_{L S} \ll \Gamma_{c}$, though.

In addition, let us place four atoms in the four special points of maximum $\Gamma_{c}$ in a subradiant state. Because the matrix of Lamb-shift couplings $\lambda_{L S}\left(\vec{r}_{i}, \vec{r}_{j}\right)$ (with $\left.\lambda_{L S}\left(\vec{r}_{i}, \vec{r}_{i}\right)=\Omega_{L S, i}\right)$ has the same signs and structure as the decay rate matrix $\Gamma\left(\vec{r}_{i}, \vec{r}_{j}\right)$, it is easy to show that it does not perturb dark states [9]. This is because dark states are eigenstates of the decay rate matrix with eigenvalue 0 , and thus also of the Lamb-shift matrix.

Although we cannot prove in general that the shape of both matrices coincides, we have observed it both in the infinite- and finite-lattice cases (Figs. 2, 3, 4, 12, and others not shown), and can be understood as follows: The LS and dissipative contributions are the integral of plane waves of all momenta with different weighting functions, see Eqs. (3) and (4). Such functions, for moderately long times, contribute mostly "very close to" and "exactly at" the resonant frequency $\Omega$ (whence the principal value and $\delta$ functions in the $t \rightarrow \infty$ limit), and thus are mediated by approximately the same manifold of excitations, having thus directional character and very similar properties. It is thus no surprise that their spatial shape is almost the same. In the case of 1D optical waveguides, the LS and dissipative terms are just the real and imaginary parts of a single exponential $\exp \left[-i k_{\Omega}\left(r_{1}-r_{2}\right)\right]$ (at the resonant frequency), and thus one cancels at atom distances where the other is maximal. In 2D though, a continuous manifold of waves contributes nontrivially, and thus 1D phenomenology is not exportable to $2 \mathrm{D}$.

\section{Entanglement decay: Perturbative vs exact}

We finish by comparing the validity of our master equation with time-dependent coefficients and the exact dynamics of the model in the one-excitation sector $[9,31]$. Take two atoms in the configuration of Fig. 12, at positions $\vec{r}_{1}=(25,20)$, $\vec{r}_{2}=(25,30)$, with initial Bell states $\left|\psi_{ \pm}\right\rangle$. Since at those points $\Gamma_{c} / \Gamma_{0}=+1$, we should, and do, observe no decay of the concurrence for $\left|\psi_{-}\right\rangle$and fast decay for $\left|\psi_{+}\right\rangle$. While the shapes are not exactly the same, the master equation faithfully captures the time it takes for the cross talk to build up. During this time, both Bell states decay because $\Gamma_{c}$ has not yet reached its final value. This time period cannot be described by the time-independent (long-time limit) Markovian equation. From 


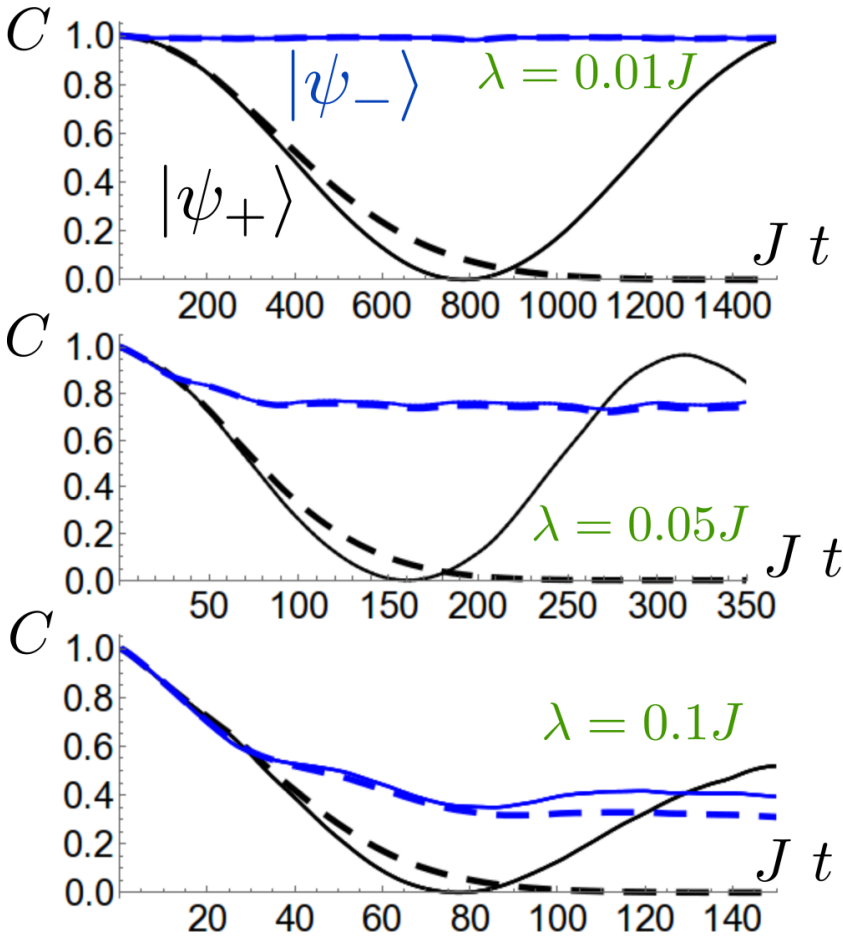

FIG. 13. Dynamics of concurrence of initial states $\left|\psi_{-}\right\rangle$blue (gray) and $\left|\psi_{+}\right\rangle$black, with $\Omega$ at the middle of the band, as in Fig. 12 (see main text). Continuous lines represent the exact dynamics, while dashed lines are the predictions of our time-dependent master equation. The system-lattice coupling is $\lambda / J=0.01$ (top), $\lambda / J=$ 0.05 (middle), and $\lambda=0.1 \mathrm{~J}$ (bottom). In the middle and bottom cases, a build-up time for the cross talk is clearly observed.

that time on, only one of the Bell states decays further. This time can be estimated as follows: In the diagonal propagation the waves have a group a velocity $\sim J$, so in order to reach one emitter from the other, they travel $r \approx 80$ lattice sites (notice that the waves follow the bouncing path in Fig. 12), thus taking $J$ as the unit, and the time is $J t \approx 80$. This can be perfectly seen in Fig. 13. If we would assume naively a decay of dark state entanglement as $C(t) \approx C(0) e^{-\lambda^{2} t}$, we obtain $0.99,0.82,0.45$ for the cases in Fig. 13 (top, middle, bottom), rather close to reality. In any given experimental realization, we should basically check the time it takes for signals to reach between emitters $t \approx r / J$ and compare to the time it takes for emitters to decay $t \approx 1 / \lambda^{2}$. If the latter is much larger, then we can safely use the long-time Markov master equation.

It is also interesting to see that once the concurrence of $\left|\psi_{+}\right\rangle$ has fallen to zero, it can never go up. This is a clear effect of the Born approximation, which takes the state of the lattice to always be the $T=0$ thermal state (vacuum). Because of this, no excitation can ever go back to the atoms. We also observe that our master equation underestimates decay, as compared to the exact dynamics which gives oscillatory behavior (revivals).
Finally, for system-lattice strong coupling $\lambda=0.1 J$ we start observing more pronounced differences in the dynamics. This is a very interesting subject for future studies.

\section{E. Experimental issues}

Apart from the finite-time propagation issue when constructing dark states in an experiment, there are other possible issues to be taken into account, such as disorder in the position of emitters. This would lead to imperfect subradiance and thus a finite-time lived dark state. In cold atoms and trapped ion configurations, there cannot be any disorder in the position of emitters, because emitters are related to degrees of freedom of the "sites" themselves. In particular for ion-trap experiments, it would be interesting to analyze the existence of directional phononic emission, since the geometry of the lattice is triangular but with a nonhomogeneous lattice spacing (more compressed near the center of the Coulomb crystal). In photonic crystal configurations, optical traps for atoms that propagate through the crystal can be used [7]. The trapping positions would very well coincide with holes bored in the dielectric material, which also coincide with the lattice "sites" for guided modes, except for finite temperature displacements of atoms from their equilibrium positions. More problematic would be issues related to emission losses, already analyzed in [32], which need to be addressed on a case-by-case basis.

\section{CONCLUSIONS}

We have studied the interaction of two probes induced by a 2D structured periodic environment. The physics of this problem is generic enough that it can be applied to, e.g., a 2D Coulomb crystal of trapped ions [1-3], PCs with nearby trapped atoms [4-7], 3D printed photonic circuits [25,26], trapped ions near piezoelectric substrates [10], or even circuit QED architectures [27-30], requiring only translational invariance of the lattice and weak system-lattice coupling. Coherent (Lamb-shift) and incoherent (radiative) couplings are studied both for finite and infinite lattices, analyzing many details and possible pitfalls. We show the usefulness of the master equation description, in terms of radiative couplings between quantum units, by showing that it directly translates into the behavior of entanglement decay of two units. Further, for more than two units, we show how to use the radiative coupling matrix to build dark states.

\section{ACKNOWLEDGMENTS}

We acknowledge fruitful discussions with A. GonzálezTudela and J. I. Cirac. This work has been supported by the EU through the H2020 Project QuProCS (Grant Agreement No. 641277), by MINECO/AEI/FEDER through Projects NoMaQ FIS2014-60343-P, QuStruct FIS2015-66860-P, and EPheQuCS FIS2016-78010-P. F.G. acknowledges funding from Vicerectorat d'Investigació i Postgrau of the UIB.
[1] S. Mavadia, J. F. Goodwin, G. Stutter, S. Bharadia, D. R. Crick, D. M. Segal, and R. C. Thompson, Nat. Commun. 4, 2571 (2013).
[2] J. W. Britton, B. C. Sawyer, A. C. Keith, C.-C. J. Wang, J. K. Freericks, H. Uys, M. J. Biercuk, and J. J. Bollinger, Nature (London) 484, 489 (2012). 
[3] J. G. Bohnet, B. C. Sawyer, J. W. Britton, M. L. Wall, A. M. Rey, M. Foss-Feig, and J. J. Bollinger, Science 352, 1297 (2017).

[4] A. Goban, C.-L. Hung, S.-P. Yu, J. D. Hood, J. A. Muniz, J. H. Lee, M. J. Martin, A. C. McClung, K. S. Choi, D. E. Chang, O. Painter, and H. J. Kimble, Nat. Commun. 5, 3808 (2014).

[5] A. Goban, C.-L. Hung, J. D. Hood, S.-P. Yu, J. A. Muniz, O. Painter, and H. J. Kimble, Phys. Rev. Lett 115, 063601 (2015).

[6] J. S. Douglas, H. Habibian, C.-L. Hung, A. V. Gorshkov, H. J. Kimble, and D. E. Chang, Nat. Photonics 9, 326 (2015).

[7] A. González-Tudela, C. L. Hung, D. E. Chang, J. I. Cirac, and H. J. Kimble, Nat. Photonics 9, 320 (2015).

[8] C. L. Hung, A. González-Tudela, J. I. Cirac, and H. J. Kimble, Proc. Natl. Acad. Sci. U.S.A. 113, E4946 (2016).

[9] F. Galve and R. Zambrini, arXiv:1711.08399.

[10] M. J. A. Schuetz, E. M. Kessler, G. Giedke, L. M. K. Vandersypen, M. D. Lukin, and J. I. Cirac, Phys. Rev. X 5, 031031 (2015).

[11] A. C. Wilson, Y. Colombe, K. R. Brown, E. Knill, D. Leibfried, and D. J. Wineland, Nature (London) 512, 57 (2014).

[12] M. Brownnutt, M. Kumph, P. Rabl, and R. Blatt, Rev. Mod. Phys. 87, 1419 (2015).

[13] F. Galve, J. Alonso, and R. Zambrini, Phys. Rev. A 96, 033409 (2017).

[14] D. A. Hite, Y. Colombe, A. C. Wilson, D. T. C. Allcock, D. Leibfried, D. J. Wineland, and D. P. Pappas, MRS Bull. 38, 826 (2013).

[15] R. H. Dicke, Phys. Rev. 93, 99 (1954).

[16] M. Gross and S. Haroche, Phys. Rep. 93, 301 (1982).

[17] R. Blume-Kohout, H. K. Ng, D. Poulin, and L. Viola, Phys. Rev. Lett. 100, 030501 (2008).

[18] J. P. Paz and A. J. Roncaglia, Phys. Rev. Lett. 100, 220401 (2008).

[19] F. Galve, A. Mandarino, M. G. A. Paris, C. Benedetti, and R. Zambrini, Sci. Rep. 7, 42050 (2017).

[20] H. J. Kimble, Nature (London) 453, 1023 (2008).

[21] S. Perseguers, M. Lewenstein, A. Acín, and J. I. Cirac, Nat. Phys. 6, 539 (2010).

[22] L.-M. Duan, M. D. Lukin, J. I. Cirac, and P. Zoller, Nature (London) 414, 413 (2001).

[23] B. Casabone, K. Friebe, B. Brandstätter, K. Schüppert, R. Blatt, and T. E. Northup, Phys. Rev. Lett. 114, 023602 (2015).

[24] R. Reimann, W. Alt, T. Kampschulte, T. Macha, L. Ratschbacher, N. Thau, S. Yoon, and D. Meschede, Phys. Rev. Lett. 114, 023601 (2015).

[25] A. Crespi, R. Osellame, R. Ramponi, D. J. Brod, E. F. Galvão, N. Spagnolo, C. Vitelli, E. Maiorino, P. Mataloni, and F. Sciarrino, Nat. Photonics 7, 545 (2013).
[26] A. Crespi, L. Sansoni, G. DellaValle, A. Ciamei, R. Ramponi, F. Sciarrino, P. Mataloni, S. Longhi, and R. Osellame, Phys. Rev. Lett. 114, 090201 (2015).

[27] A. A. Houck, H. E. Türeci, and J. Koch, Nat. Phys. 8, 292 (2012).

[28] A. F. van Loo, A. Fedorov, K. Lalumi'ere, B. C. Sanders, A. Blais, and A. Wallraff, Science 342, 1494 (2013).

[29] Y. Liu and A. A. Houck, Nat. Phys. 13, 48 (2017).

[30] M. Fitzpatrick, N. M. Sundaresan, A. C. Y. Li, J. Koch, and A. A. Houck, Phys. Rev. X 7, 011016 (2017).

[31] A. González-Tudela and J. I. Cirac, Phys. Rev. Lett. 119, 143602 (2017).

[32] A. González-Tudela and J. I. Cirac, Phys. Rev. A 96, 043811 (2017).

[33] L. van Hove, Phys. Rev. 89, 1189 (1953).

[34] N. W. Ashcroft and N. D. Mermin, Solid State Physics (Harcourt College Publishers, San Diego, CA, 1976).

[35] H. P. Breuer and F. Petruccione The Theory of Open Quantum Systems (Oxford University Press, Oxford, 2003).

[36] C. Benedetti, F. Galve, A. Mandarino, M. G. A. Paris, and R. Zambrini, Phys. Rev. A 94, 052118 (2016).

[37] S. Diehl, A. Micheli, A. Kantian, B. Kraus, H. P. Büchler, and P. Zoller, Nat. Phys. 4, 878 (2008).

[38] M. Morse, Trans. Amer. Math. Soc. 27, 345 (1925).

[39] S. John and J. Wang, Phys. Rev. Lett. 64, 2418 (1990).

[40] S. John and T. Quang, Phys. Rev. A. 50, 1764 (1994).

[41] F. Galve and R. Zambrini, Int. J. Quantum Inf. 12, 1560022 (2014).

[42] T. Shi, Y.-H. Wu, A. González-Tudela, and J. I. Cirac, Phys. Rev. X 6, 021027 (2016).

[43] G. Calajó, F. Ciccarello, D. Chang, and P. Rabl, Phys. Rev. A 93, 033833 (2016).

[44] A. Mekis, M. Meier, A. Dodabalapur, R. Slusher, and J. Joannopoulos, Appl. Phys. A 69, 111 (1999).

[45] A. H. Castro Neto, F. Guinea, N. M. R. Peres, K. S. Novoselov, and A. K. Geim, Rev. Mod. Phys. 81, 109 (2009).

[46] J. Eisert and M. B. Plenio, Int. J. Quant. Inf. 1, 479 (2003).

[47] G. Adesso and F. Illuminati, J. Phys. A 40, 7821 (2007).

[48] S. Olivares, Eur. Phys. J. Spec. Top. 203, 3 (2012).

[49] W. K. Wootters, Phys. Rev. Lett. 80, 2245 (1998).

[50] G. Vidal and R. F. Werner, Phys. Rev. A 65, 032314 (2002).

[51] J. D. Joannopoulos, S. G. Johnson, J. N. Winn, and R. D. Meade, Photonic Crystals: Molding the Flow of Light (Princeton University Press, Princeton, NJ, 2007).

[52] S. Fan and J. D. Joannopoulos, Phys. Rev. B 65, 235112 (2002).

[53] J. O. Grepstad, M. M. Greve, B. Holst, I.-R. Johansen, O. Solgaard, and A. Sudbø, Opt. Express 21, 23640 (2013). 